\title{
Hemodynamic Response to Volume Stress in Awake Late-Pregnant and Nonpregnant Rats
}

\author{
PETER F. BOEKKOOI, BEN J. A. JANSSEN, CARLA M. VERKESTE, PETER KAUFMAN, AND \\ LOUIS L. H. PEETERS \\ Department of Obstetrics and Gynecology [P.F.B., C.M.V., L.L.H.P.] and Department of Pharmacology \\ [B.J.A. J.]. University of Limburg, Maastricht, The Netherlands, and Department of Anatomy, Rheinisch \\ Westfalische Technische Hochschule, Aachen, Germany [P.K.]
}

\begin{abstract}
The present study was designed to determine whether the often observed gestational rise in blood volume alters the hemodynamic response to volume stress relative to the nonpregnant state. Late-pregnant and nonpregnant Wistar rats, prepared with an electromagnetic flow probe around the ascending aorta and/or intravascular catheters were used for this study. One of the following sets of measurements were performed either before and after volume loading or before and after volume depletion $(24 \mathrm{~h}$ thirst): 1) continuous monitoring of cardiac output, blood pressure, heart rate, and fluid balance (only during volume loading); 2) measurement of cardiac output and regional blood flows (microspheres); and 3) measurement of the inferior vena cava diameter ( $\mathrm{x}$-ray). Both rapid (bolus) and slow volume loading (infusion) led to a rise in cardiac output. The generated surplus in cardiac output was distributed to the kidneys, skeletal tissues, and intestines (only in nonpregnant rats), and was paralleled in the pregnant rats by a consistent fall in placental blood flow. Slow volume depletion had neither a consistent effect on cardiac output nor on its distribution, with even a preserved maturational rise in placental blood flow during the $24 \mathrm{~h}$ of thirst. Slow volume loading and depletion led to an increase and decrease of the caliber of the inferior vena cava, respectively. The nonnuterine hemodynamic response to all forms of volume stress had not changed in pregnancy. The results of this study suggest that the hemodynamic response to volume stress consists of three components: $I$ ) a venous response that dampens with a certain lag time the effects of volume changes on venous return; 2) an instantaneous response of the volume regulatory system, which includes a change in renal perfusion; and 3) an effect on venous return and, with it, cardiac output that only develops when the veins are unable to buffer imposed volume changes. If the latter causes systemic flow to increase in excess of nutritional demands, the surplus flow is shunted through small arteriovenous shunts in skeletal tissues (and intestines in nonpregnancy), to protect the systemic capillary bed. Massive systemic shunting on top of an already congested venous compartment can be expected to raise mean circulatory filling pressure. It is speculated that the latter causes the selective decrease in placental flow after volume loading through a direct negative effect on the peculiar (rat) placental microcirculation. (Pediatr Res 30: 479-486, 1991)
\end{abstract}

\section{Abbreviations}

IVC, inferior vena cava

MCFP, mean circulatory filling pressure

Received August 16, 1990; accepted June 21, 1991

Correspondence and reprint requests: Louis L. Peeters, M.D., Department of Obstetrics and Gynecology, Academic Hospital Maastricht, PO Box 5800, 6202 AZ Maastricht, The Netherlands.

\author{
$\mathrm{BV}$, blood volume $(\mathrm{mL})$ \\ $\mathrm{CO}$, cardiac output $(\mathrm{mL} / \mathrm{min})$ \\ HR, heart rate (beats $/ \mathrm{min}$ ) \\ $\mathrm{SV}$, stroke volume $(\mu \mathrm{L})$ \\ MAP, mean arterial pressure (torr) \\ SVR, systemic vascular resistance $(\mathrm{mm} \mathrm{Hg} \cdot \mathrm{min} / \mathrm{mL})$ \\ Hct, hematocrit (vol\%) \\ $\mathrm{RBC}$, red blood cell \\ $P$, pregnant \\ NP, nonpregnant \\ Th, thirsting \\ Nonth, nonthirsting \\ exp, experimental \\ contr, control \\ rat $_{\text {exp }}$, experimental rat \\ rat $_{\text {contr, }}$ control rat \\ $\mathbf{A} \leftrightarrow \mathbf{P}$, anterior-posterior
}

Both maternal $\mathrm{CO}$ and $\mathrm{BV}$ begin to increase in early human pregnancy to reach a plateau of $\approx 30 \%$ above nonpregnant values by the 16th and 34th wk, respectively (1-4). However, since BV and $\mathrm{CO}$ have never been measured together throughout pregnancy, their precise interrelation in pregnancy is still obscure. In the nonpregnant state, $\mathrm{BV}$ and $\mathrm{CO}$ vary as a function of each other, if either one is changed acutely (5-7). In pregnancy, $\mathrm{CO}$ and $\mathrm{BV}$ increase gradually over a period of weeks to months (14), a condition that may be associated with an altered response of the volume regulatory system. Insight in the latter in pregnancy has become particularly important after the introduction in recent years of aggressive volume expansion therapy in the symptomatic management of the preeclamptic patient $(8,9)$, a development that evolves in spite of still almost complete lack of understanding of the impact on BV regulation of the dynamic state of pregnancy.

The objective of the present study in the rat was to test the hypothesis that the gradually expanding BV compartment in normal pregnancy does not alter the hemodynamic response to an imposed volume load or volume depletion. The impact of pregnancy was determined by comparing the hemodynamic response induced by volume loading/volume depletion in pregnancy with that observed in the NP state. Besides the magnitude, also the rate by which volume stress is imposed can be expected to influence the hemodynamic response $(10,11)$. Therefore, volume loading was implemented at different rates. Volume depletion was only induced slowly by 24 -h thirsting in an attempt to limit the interference with the response of a concomitant rise in sympathetic tone. The awake Wistar rat was considered a suitable model for this study, as BV in late pregnancy in this animal is about $40 \%$ higher than in the NP state (12). 


\section{MATERIALS AND METHODS}

Validations. The difference in baseline BV between the 19-d $P$ and NP Wistar rats in the present study was determined as follows. Seven P and six NP rats were anesthesized with ether, and polyethylene catheters (PE-10, outer diameter $=0.61 \mathrm{~mm}$, inner diameter $=0.28 \mathrm{~mm}$, heat-sealed to a piece of PE-50 tubing, outer diameter $=0.92 \mathrm{~mm}$, inner diameter $=0.58 \mathrm{~mm}$ ) were inserted into the abdominal aorta and IVC and advanced from a femoral artery and vein, respectively. The next day the animals received an i.v. bolus injection of $1 \mu \mathrm{Ci}{ }^{25} \mathrm{I}$-albumin dissolved in $0.5 \mathrm{~mL}$ saline. Plasma sp act was calculated on the basis of extrapolation to time zero of the radioactivity determined in $0.2-\mathrm{mL}$ blood samples collected after $5,10,15$, and $20 \mathrm{~min}$. BV was derived by multiplying plasma volume with $1 /(1-\mathrm{Hct})$.

In the present study, BV changes were provoked by subjecting awake catheterized NP and $P$ rats to various regimens of volume loading or volume depletion. The induced changes in BV were determined from the observed change in Hct. In our study, this approach was preferred over standard indicator dilution techniques, to avoid the use of radioactivity and to minimize blood sampling. Because this approach is unconventional, it was considered appropriate to validate this procedure beforehand. Four $P$ rats provided with catheters in the femoral artery and vein on the preceding day were subjected to $2 \mathrm{~h}$ volume loading with Haemaccel (Hoechst, Germany) at a rate of $6 \mathrm{~mL} / \mathrm{h}$. Before and again after volume loading, $0.8 \mathrm{~mL}$ of a cocktail containing ${ }^{51} \mathrm{Cr}$ labeled RBC and ${ }^{125} \mathrm{I}$-albumin was injected i.v. Red cell volume and plasma volume were calculated as described previously (13). Before the 2nd cocktail injection, an arterial sample was withdrawn to determine background radioactivity. The radioactivity in the 2 nd cocktail was three times as high as that in the 1st cocktail. Before the 1st cocktail and again together with the last blood sampling, $0.05 \mathrm{~mL}$ extra blood was withdrawn to determine the Hct.

Confirmation of stability in fluid balance after slow volume loading. Hemodynamic steady state including stable fluid balance at the time of measurement was considered a prerequisite for the reliable measurement of organ flows with microspheres in this study. Therefore, we confirmed in a separate group of three previously catheterized 19-d P rats that the stability in HR and MAP after $2 \mathrm{~h}$ of slow volume loading applied also to the fluid balance. In these animals, the cumulative urine output and Hct was determined at 30-min intervals, and MAP and HR were monitored continuously throughout $5 \mathrm{~h}$ of infusion with Haemaccel at a rate of $6 \mathrm{~mL} / \mathrm{h}$.

Experiment 1. Experiment 1 was performed in six 19-d $\mathrm{P}$ and six age-matched virgin NP Wistar rats obtained from a local breeder (HSD, Zeist, The Netherlands). The rats (P, 14th d of pregnancy) were anesthetized with ketamin $(20 \mathrm{mg})$ and xylazine (4 mg) intramuscularly, and the trachea was intubated (PE-240). After establishing positive pressure respiration $(60$ breaths $/ \mathrm{min}$, tidal volume $2.5-3 \mathrm{~mL}$ ), the surgery was carried out under aseptic conditions. The skin and muscle overlying the $3 \mathrm{rd}$ right intercostal space were incised and separated. The 3rd and 4th rib were spread and the ascending aorta was freed from its surrounding tissue. An electromagnetic flow probe (outer diameter $=2.3$ $\mathrm{mm}$; Skalar, Delft, Holland) was fitted around the ascending aorta just above the heart. The thorax was closed and the probe cable fixed to the ribs. The cable was guided to the neck where the connector was exteriorized. The surgical procedure has been described in detail elsewhere (14). Four d after the probe implantation (in $\mathrm{P}$ rats, $\mathrm{d} 18$ of pregnancy) the rats were prepared with an abdominal and an IVC catheter as detailed above. The catheters were tunneled s.c. to the same exit site as the probe cable. After surgery, the rats were placed in a metabolic cage to habituate them to the subsequent experimental environment.

On the next day ( $\mathrm{P}$ rats, 19 th $\mathrm{d}$ of pregnancy), the rats were placed in metabolic cages $(22 \times 13 \times 13 \mathrm{~cm})$ and connected to the measuring equipment. The flow probe was connected to a sine-wave electromagnetic flow meter (Skalar), and the arterial catheter, to a pressure transducer. Data were recorded continuously and stored on a hard disk of a real-time data processing system (Dept. of Instrumental Services, Faculty of Medicine, University of Limburg, Maastricht, The Netherlands). This system has virtually no drift because it corrects for baseline before each beat, taking late-diastolic flow as zero flow. Linearity and accuracy of the system were $1 \%$ and less than $7 \%$, respectively (14). After a stabilization period of at least $45 \mathrm{~min}$, recording of the hemodynamic variables was started. CO, HR, and MAP were monitored continuously. In NP rats, measurements were continued until $30 \mathrm{~min}$ after discontinuation of volume loading. SV and SVR were calculated from CO, HR, and MAP. After the experiments, the animals were killed (overdose of pentobarbital) and, in $\mathrm{P}$ rats, the fetuses counted and weighed.

The experiment consisted of an i.v. bolus injection $(2 \mathrm{~mL})$ immediately followed by a 2 - $\mathrm{h}$ infusion with the plasma expander Haemaccel at a rate of $6 \mathrm{~mL} / \mathrm{h}$. Haemaccel is an isotonic colloid solution composed of a polymer of urea and polypeptides. It is readily excreted by the kidneys with minimal metabolic breakdown. During infusion, the agent remains within the intravascular compartment with no measurable leakage to the interstitium (15). Haemaccel is known to be well tolerated by the rat with no appreciable effect on MAP, HR, and respiratory rate, even when infused in large amounts (16). It does not alter serum concentrations of electrolytes. Because of the latter and the isotonicity of the solution, the effect of Haemaccel on red cell volume is negligible. The infusion rate of $6 \mathrm{~mL} / \mathrm{h}$ was chosen on the basis of previous testing, which indicated that a lower infusion rate had an inconsistent effect on $\mathrm{BV}$, whereas a more rapid infusion rate often failed to result in a stable fluid balance within $2 \mathrm{~h}$.

Experiment 2. In seven 19-d P and six NP Wistar rats, the distribution of the $\mathrm{CO}$ was determined before and after the $2 \mathrm{~h}$ slow volume loading as detailed above. Two $d$ before the experiment, the rats were anesthetized with ether, and polyethylene catheters with dimensions as detailed above were inserted into the right carotid artery, a femoral artery, and a femoral vein and advanced into the left ventricle of the heart, the abdominal aorta, and the IVC, respectively. The catheters were tunneled s.c. and exteriorized between the shoulder blades. The day before the experiment, also these rats were allowed to adapt to the metabolic cages in which the experiment was to be performed.

$\mathrm{CO}$ and its distribution were measured with $15-\mu \mathrm{m}$ radioactive microspheres using the reference sampling technique (17). To this end, approximately $1.2 \times 10^{5}$ microspheres (NEN, Dreieich, Germany), suspended in $0.2 \mathrm{~mL}$ saline with Tween $80(0.05 \%)$ added to prevent aggregation, were injected into the left ventricle over a period of $15 \mathrm{~s}$ while a reference sample was withdrawn from the abdominal aorta at a rate of $0.5 \mathrm{~mL} / \mathrm{min}$. Reference sampling encompassed the period between $10 \mathrm{~s}$ before the microspheres injection and $30 \mathrm{~s}$ after its completion. The blood withdrawn was replaced by fresh heparinized blood from a donor Wistar rat. After $2 \mathrm{~h}$ of slow volume loading, the microspheres experiment was repeated using a different radioactive label.

After the 2nd microspheres experiment, the animals were killed and all organs were dissected and weighed. The radioactivity in each tissue aliquot and reference sample was determined with a sodium crystal scintillation counter (Packard, Delft, The Netherlands). CO was obtained by multiplying the ratio of total $\mathrm{cpm}$ in the body and cpm in the reference sample by the reference sampling rate. Organ flows were calculated by multiplying fractional entrapment of microspheres in each organ with the reference sampling rate (18). In all rats mixing of the microspheres was considered adequate on the basis of a difference of less than $5 \%$ in fractional entrapment of microspheres by the two kidneys (19). The reference sample always contained 400-700 microspheres.

Experiment 3. Experiment 3 involved the determination of the impact of volume loading on the degree of filling of the IVC. 
Six pairs of NP and fours pairs of 19-d P Wistar rats were anesthetized with ether and pair-wise positioned in dorsal recumbency on a heated pad. In each rat, a catheter (PE-10) was inserted into a femoral vein and advanced until just below the outlet into the IVC. One rat in each pair was subjected to volume loading ( rat $_{\text {exp }}$ ) and its counterpart served as control (rat contr $_{\text {) }}$. After a stabilization period of $20 \mathrm{~min}$ (baseline), $1 \mathrm{~mL}$ of contrast medium (Hexabrix) was rapidly infused i.v. in both rats simultaneously, using an infusion pump at a constant rate of $6 \mathrm{~mL} /$ min, over a period of $10 \mathrm{~s}$. Then, an $\mathrm{A} \leftrightarrow \mathrm{P}$ and a lateral $\mathrm{x}$-ray were taken of both rats laying next to each other. After completion of the lateral $x$-ray, the rats were repositioned in dorsal recumbency and a $2-\mathrm{mL}$ bolus injection of Haemaccel was only given to the rat ${ }_{\text {exp }}$. Immediately after the bolus, a second set of $\mathrm{x}$-rays was taken using the same standardized procedure. Then, a steady state infusion with Haemaccel at a rate of $6 \mathrm{~mL} / \mathrm{h}$ was started in the ratexp. Two h later, the procedure to take $\mathrm{x}$-rays of the rat pair was repeated.

The difference in IVC diameter was determined as follows. On each $x$-ray the IVC diameter was determined by taking the mean of three measurements at three distinct points in the IVC between the bifurcation and the hepatic venous outlet. In the six NP rats $_{\mathrm{cxp}}$ and four $\mathrm{P}$ rats $\mathrm{exp}_{\mathrm{ex}}$, the IVC diameter after rapid and slow volume loading was compared with the previously measured baseline IVC diameter (intraanimal comparison). The possible contribution of random and/or systematic error to the results was determined in the control rats from the within-animal difference in IVC between the three measurement sessions.

Experiment 4 . The placentas from six 19-d P rats were studied morphologically. Three of these rats were subjected to slow volume loading as detailed above and three served as controls. The experimental rats were killed immediately after completion of volume loading. The placentas of all viable fetuses were weighed and incubated for $3 \mathrm{~d}$ in buffered $4 \%$ formaldehyde, mounted in paraffin, sliced, and stained using standard laboratory techniques. An experienced placentologist (co-author P.K.) judged the placental slides by light microscopy, without foreknowledge on preceding exposure to volume load.

Experiment 5. Experiment 5 was designed to quantify the changes in BV and urine output in response to 24-h thirsting and was performed on eight 19-d P and eight NP Wistar rats. The induced fall in BV was calculated from the rise in Hct. Hct was measured in duplicate (microcapillary technique) in a blood sample $(25 \mu \mathrm{L})$ obtained from the orbital venous plexus before and after 24-h thirsting. Blood sampling was performed while the animal was briefly sedated with ether. The impact of thirsting on the fluid balance was determined by measuring weight loss, water intake, and urine output during a 24-h control period and during the subsequent 24-h thirsting.

Experiment 6 . Experiment 6 was designed to determine the hemodynamic response to $24-\mathrm{h}$ thirsting in six 19-d P (ThP/exp) and seven NP rats (ThNP/exp). To separate the effects caused by thirsting from those associated with ongoing pregnancy, the hemodynamic changes in the ThP/exp group were compared with those observed in a group of seven 19-d P rats (NonthP/ contr) that had unlimited access to water throughout the experimental period. Two $\mathrm{d}$ before the experiment, the rats were anesthetized with ether and prepared with a left ventricle and a femoral artery catheter as described for experiment 2 . In the postsurgery recovery period, the rats were kept in metabolic cages to habituate them to the actual experimental environment. Then, measurements in baseline (in ThP/exp and NonthP/contr: $\mathrm{d} 19$ of pregnancy) and after 24-h thirsting (ThP/exp) or nonthirsting (NonthP/contr) were performed. MAP and HR were monitored continuously during both measurement sessions until after completion of the blood flow measurement. $\mathrm{CO}$ and organ flows were determined with $15-\mu \mathrm{m}$ microspheres after a 40 -min stabilization period, as described for experiment 2.

Experiment 7. Experiment 7 involved the evaluation of the impact of 24-h thirsting on the diameter of the IVC. Three pairs of NP and four pairs of 19-d P rats were anesthetized with ether and pair-wise positioned in dorsal recumbency on a heated pad. The day before measurement, the rats were prepared with two femoral vein catheters. One rat in each pair was subjected to 24 $\mathrm{h}$ thirsting while the (control) counterpart had free access to water. After a 20-min stabilization period and again the next day, x-rays were taken according to the standardized protocol described above (experiment 3).

Data are presented as means \pm SD throughout the text. Differences between control and experimental observations were evaluated by the Wilcoxon signed rank test. Differences between $P$ and NP groups and differences between the changes in the $\mathrm{ThP} / \mathrm{exp}$ group and the ThP/contr were evaluated with the Mann-Whitney test. A probability of less than $5 \%$ (two-sided) was considered significant.

\section{RESULTS}

Validations. BV was about $25 \%$ higher in $\mathrm{P}(23.3 \pm 2.2 \mathrm{~mL})$ than in NP rats $(18.9 \pm 1.8 \mathrm{~mL})$. The induced BV increase in the validation study ranged from 28 to $64 \%$. Despite this large variation, the increase in BV calculated from the fall in Hct and that simultaneously determined with the indicator dilution technique differed by only $1-4 \%$ ( 28 versus $31 \% ; 45$ versus $41 \%, 35$ versus $34 \%$, and 64 versus $68 \%$, respectively).

The fluid balance during $5 \mathrm{~h}$ of slow volume loading at $6 \mathrm{~mL} /$ $\mathrm{h}$ is illustrated for one rat in Figure 1. In all rats urine output accelerated within $\approx 30 \mathrm{~min}$ and always equilibrated with volume input within $2 \mathrm{~h}$. In steady state the total amount of infused Haemaccel exceeded the cumulative urine output by $\approx 9 \mathrm{~mL}$.

Experiment 1. Maternal weight in the P group $(277 \pm 31 \mathrm{~g})$ was higher than in the NP group $(236 \pm 52 \mathrm{~g})$. The litter size was $6 \pm 1$ with fetuses weighing $1.6 \pm 0.2 \mathrm{~g}$. Table 1 lists various central hemodynamic variables in baseline, after rapid and after slow volume loading in $\mathrm{P}$ and NP rats. In $\mathrm{P}$ rats baseline HR was higher and baseline MAP and SVR lower than in NP rats. Table 2 lists the percentage of change in these parameters after both forms of volume loading. In both $\mathrm{P}$ and NP rats, rapid volume load increased $\mathrm{CO}$ and $\mathrm{SV}$ by $\approx 30 \%$ and MAP by $\approx 5 \%$. SVR had decreased by $\approx 15 \%$, and HR had not changed appreciably. The changes induced by slow volume loading were almost identical, except for the normalization of the MAP and the fall in HR in the $\mathrm{P}$ rats only. Figure 2 illustrates for both groups the patterns in these variables throughout slow volume loading. All appeared to have reached steady state after $1 \mathrm{~h}$ despite ongoing fluid accumulation in the subsequent hour (Fig. 1). The small (in P) or absent (in NP) change in HR implies that the rise in $\mathrm{CO}$ in response to both forms of volume loading was almost entirely accomplished by a rise in SV. Although the mean rise in

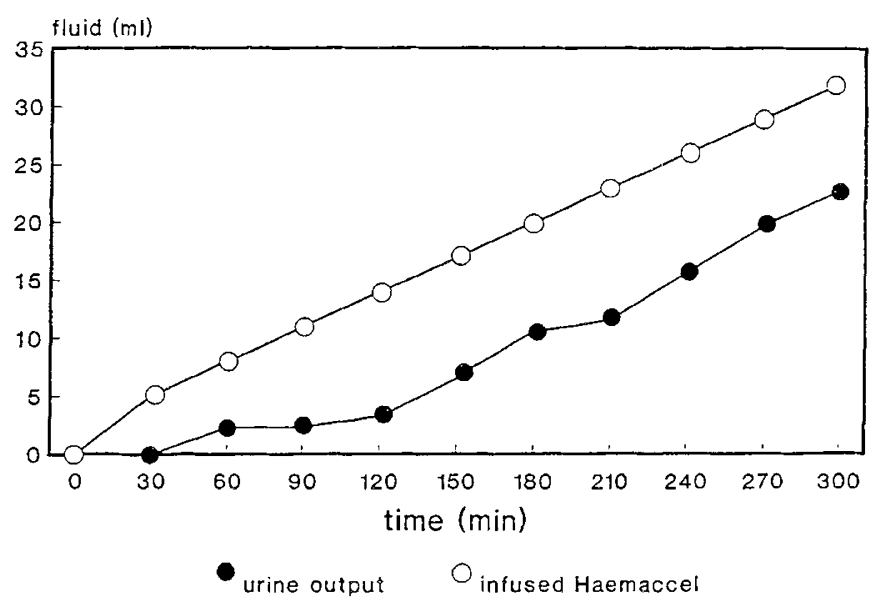

Fig. 1. Cumulative volume input and urine output throughout a 5 -h period of volume loading at $6 \mathrm{~mL} / \mathrm{h}$, in a $19-\mathrm{d} \mathrm{P}$ rat. 
Table 1. Hemodynamic parameters in baseline, after rapid volume loading $(V L)$, and after slow volume loading in $P(n=6)$ and $N P(n=6)$ rats

\begin{tabular}{|c|c|c|c|c|c|c|}
\hline & \multicolumn{3}{|c|}{$\mathrm{P}$} & \multicolumn{3}{|c|}{ NP } \\
\hline & Baseline & Rapid VL & Slow VL & Baseline & Rapid VL & Slow VL \\
\hline $\mathrm{CO}(\mathrm{mL} / \mathrm{min})$ & $88 \pm 11$ & $116 \pm 14^{*}$ & $117 \pm 12^{*}$ & $78 \pm 8$ & $105 \pm 8^{*}$ & $113 \pm 11$ \\
\hline MAP (torr) & $90 \pm 8$ & $99 \pm 12^{*}$ & $94 \pm 7$ & $115 \pm 12 \dagger$ & $121 \pm 13^{*}$ & $114 \pm 25$ \\
\hline $\mathrm{HR}(\mathrm{bpm})$ & $445 \pm 24$ & $433 \pm 22$ & $416 \pm 31^{*}$ & $383 \pm 11 \dagger$ & $405 \pm 24$ & $423 \pm 28$ \\
\hline $\mathrm{SV}(\mu \mathrm{L})$ & $201 \pm 31$ & $268 \pm 42^{*}$ & $281 \pm 29^{*}$ & $203 \pm 20$ & $259 \pm 24^{*}$ & $266 \pm 19^{*}$ \\
\hline $\mathrm{SVR}(\mathrm{mm} \mathrm{Hg} \cdot \mathrm{min} / \mathrm{mL})$ & $1.1 \pm 0.2$ & $0.9 \pm 0.2^{*}$ & $0.8 \pm 0.2^{*}$ & $1.5 \pm 0.2 \dagger$ & $1.2 \pm 0.2^{*}$ & $1.0 \pm 0.2^{*}$ \\
\hline
\end{tabular}

* Significant difference from baseline $(p<0.05)$.

$\uparrow$ Significant difference between groups $(p<0.05)$.

Table 2. Percentage of change in central hemodynamic parameters after rapid and slow volume loading $(V L)^{*}$

\begin{tabular}{lccccc}
\hline & \multicolumn{2}{c}{$\mathrm{P}$} & & \multicolumn{2}{c}{$\mathrm{NP}$} \\
\cline { 2 - 3 } \cline { 5 - 6 } & Rapid VL & Slow VL & & Rapid VL & Slow VL \\
\hline BV & & $+18 \pm 10$ & & $+20 \pm 2$ \\
CO & $+32 \pm 11$ & $+35 \pm 18$ & & $+32 \pm 12$ & $+47 \pm 18$ \\
SV & $+33 \pm 8$ & $+42 \pm 20$ & & $+29 \pm 14$ & $+32 \pm 8$ \\
HR & NS & $-2 \pm 1$ & & NS & NS \\
MAP & $5 \pm 2$ & NS & $6 \pm 2$ & NS \\
SVR & $-18 \pm 4$ & $-23 \pm 13$ & $-12 \pm 5$ & $-31 \pm 18$ \\
\hline
\end{tabular}

* All \% changes listed were significant $(p<0.05$, Wilcoxon signed rank test).

BV $(\approx 20 \%)$ in response to slow volume loading was comparable in $\mathrm{P}$ and NP rats, the interanimal variation was larger in the $\mathrm{P}$ group (Table 2). Only NP rats were monitored until 30 min after discontinuation of volume loading. In this period $\mathrm{CO}, \mathrm{SV}$, and SVR tended to return to baseline (Fig. 2).

Experiment 2. The litter size in the seven $\mathrm{P}$ animals varied between 10 and 14 fetuses. Slow volume loading induced a similar rise in $\mathrm{BV}(\approx 20 \%)$ as seen in experiment 1 . Systemic shunting (defined as the percentage of microspheres recovered from the lungs) and organ flows before and after slow volume loading are listed in Table 3 . Base line systemic shunting and all organ flows except for that to the myometrium (higher in $\mathrm{P}$ rats) were comparable in $\mathrm{P}$ and NP rats. Renal and carcass flow (skeletal muscle, bone, connective tissue) had increased in both groups. In the NP group, intestinal flow had also increased consistently, whereas in the $\mathrm{P}$ group placental blood flow had decreased consistently. The percentage of change in renal, intestinal, carcass, and placental flows in response to slow volume loading is listed in Table 4.

Experiment 3. Neither in $\mathrm{P}$ rats $\mathrm{exp}_{\mathrm{e}}$ nor in NP rats exp $_{\text {did }}$ the IVC diameter change consistently after rapid volume loading. In contrast, after slow volume loading the IVC diameter on the $\mathrm{A} \leftrightarrow \mathrm{P}$ picture had increased consistently by $24 \pm 11 \%$ and $28 \pm$ $13 \%$, respectively. Meanwhile, the concomitantly measured IVC diameter on the lateral pictures had changed inconsistently. The intrapair comparison for each experimental state yielded almost identical results, although with a higher variation. Figure 3 illustrates for one $\mathrm{P}$ rat pair the $\mathrm{A} \leftrightarrow \mathrm{P}$ IVC outline before and after slow volume loading.

An impression about the measurement error was obtained by comparing the IVC diameter as deduced from the three $\mathrm{x}$-ray

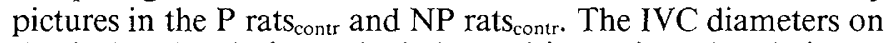
the 2 nd and 3 rd picture had changed inconsistently relative to that on the 1st picture. Because the x-ray method combined a high reproducibility with a large measurement error (coefficient of variation $=46 \%$ ), it was decided to use this method only to obtain qualitative information on induced changes in IVC diameter.

Experiment 4. The placental morphology in baseline and after slow volume loading is illustrated in Figures 4 and 5, respectively. In baseline both maternal and fetal vessels are filled with small clusters of RBC. After slow volume loading, RBC density had markedly increased in the maternal lacunas causing compression of fetal trabeculae and fetal microvessels.

Experiment 5. Table 5 lists the results obtained in experiment 5 with respect to the effects of 24-h thirsting on body weight, $\mathrm{BV}$, and fluid balance. $\mathrm{P}$ rats were heavier and had a lower baseline Hct. In both $\mathrm{P}$ and NP rats, thirsting caused a decrease in $\mathrm{BV}$ and body weight by $7 \%$ and $16-19 \mathrm{~g}$, respectively. $\mathrm{P}$ rats showed a higher daily water intake in baseline and a lower urine production during thirsting compared with the NP rats.

Experiment 6 . The central hemodynamics before and after thirsting are listed in Table 6. In neither group had CO, MAP, HR, SV, and SVR changed consistently after thirsting (ThNP and ThP/exp) or nonthirsting (NonthP/contr). Table 7 lists for both experimental states the magnitude of systemic shunting and organ flows in the three groups. The lung fraction had decreased by a similar margin in the two ThP groups. Neither in the ThP/ exp nor in the NonthP/contr group had the nonuterine organ flows changed appreciably after the $24 \mathrm{~h}$ of (non)thirsting. The maturational increase in placental blood flow observed in the NonthP/contr group was not affected by the exposure to $24 \mathrm{~h}$ of thirsting (ThP/exp).

Experiment 7 . Both $\mathrm{P}$ and NP rats responded to thirsting with a variable $(10-50 \%)$ but consistent decrease in the IVC diameter on the A $\leftrightarrow$ P pictures as illustrated for one NP rat pair (Fig. 6). The concomitant change on the lateral pictures was inconsistent.

\section{DISCUSSION}

BV expansion in pregnancy is known to be important for the uneventful development of pregnancy. However, neither purpose of the physiologic $\mathrm{BV}$ expansion nor its consequences for volume regulation are well understood. One of the consequences of the physiologic BV expansion may be an altered set point and/or gain of the volume regulatory response. In this study, adaptive changes in the volume regulatory system during pregnancy were explored by comparing the hemodynamic response to volume stress in $\mathrm{P}$ rats with that in NP rats. The 19-d P rat was chosen for these studies, as BV at $19 \mathrm{~d}$ was found to have increased $\approx 25 \%$ relative to $\mathrm{NP}$ values.

Before the actual study, we confirmed that the methods adopted to impose volume load led to stability in hemodynamics and fluid balance at the time of measurements. It was also demonstrated that induced BV changes were reliably quantified from the fall in Hct and that qualitative changes in the venous filling state could be discerned from the changes in IVC outline on x-rays.

To our knowledge, no data have been reported on the use of an electromagnetic $\mathrm{CO}$ probe in awake $\mathrm{P}$ rats. In the present study, the surgery needed to implant a CO probe was found to be associated with considerable stress to the $\mathrm{P}$ rat. This was reflected in a fetal survival rate of only $50 \%$. However, the impact of the stress appeared to be confined to the immediate postsurgical period, inasmuch as, on the 5th postsurgical day, these rats showed not only baseline hemodynamics similar to those in $\mathrm{P}$ rats without a $\mathrm{CO}$ probe (experiment 1 ; lower MAP 
CARDIAC OUTPUT

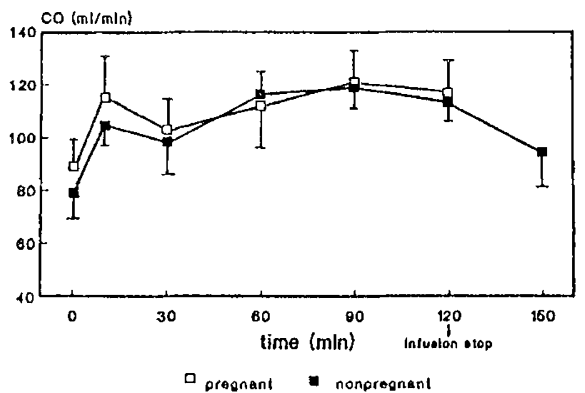

STROKE VOLUME

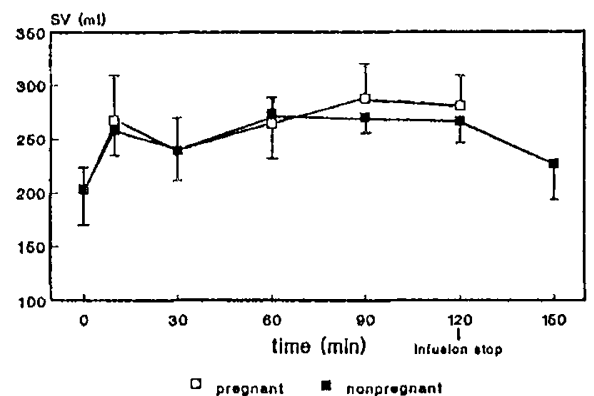

TOTAL PERIPHERAL RESISTANCE

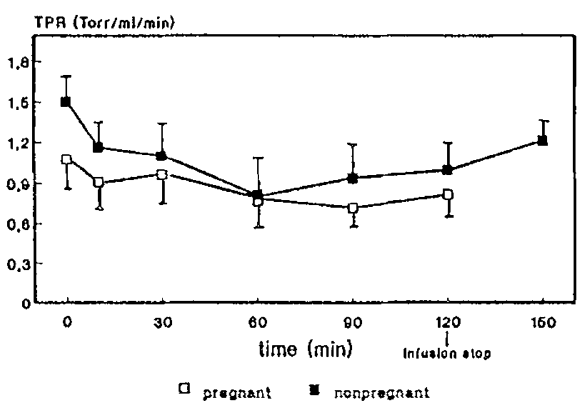

MEAN ARTERIAL PRESSURE



HEART RATE

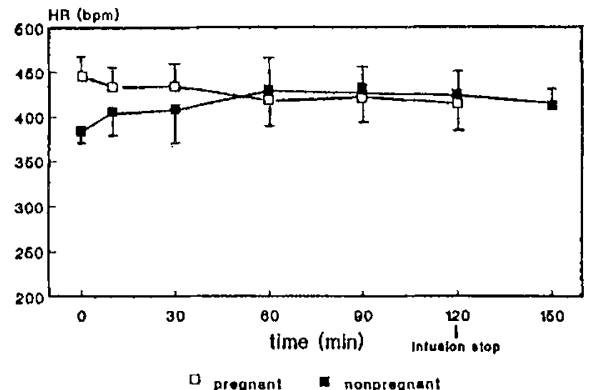

Fig. 2. Central hemodynamics during rapid and slow volume loading in P and NP rats. In the NP rats, monitoring was continued until 30 min after the end of volume loading.

and SVR), but also unaffected growth of the remaining viable fetuses.

In the present study, the volume regulatory system was challenged by imposing either volume load or volume depletion. Rapid volume loading induced an immediate $\approx 30 \%$ rise in $\mathrm{CO}$ in both groups, almost entirely accomplished by a rise in SV (Table 2). The $30 \%$ rise in $\mathrm{CP}$ was reached almost instantaneously after the bolus injection (Fig. 2) and was not accompanied by appreciable venous dilatation. This apparent venous inertia is most likely a consequence of the so-called "stressrelaxation" of the veins (11), which refers to the protracted response of the venous compliance to a rapidly imposed change in venous filling state. It may take as much as 30 min before the adapting venous compliance stabilizes at its new level, depending on basal venous filling state and rate and magnitude of the imposed volume load $(7,10,11,20,21)$. The gradual rise in venous compliance during the exposure to an increased volume is paralleled by a gradual fall in venous pressure and, with it, normalization of the initially elevated venous return. The immediate $5 \%$ rise in MAP after rapid volume loading can be expected to contribute to the normalization of the acutely elevated $\mathrm{CO}$ through activation of the baroreceptor, a mechanism supported by previous studies (22).
Rapid and slow volume loading induced a comparable rise in $\mathrm{CO}$ in $\mathrm{P}$ rats (Table 2), despite a twice as large rise in $\mathrm{BV}$ in the latter ( $18 \%$ versus $2 / 23.3 \mathrm{~mL}$ or $\approx 9 \%$ ). Assuming also comparable excess $\mathrm{CO}$ after both forms of volume loading, this result indicates that the venous dilatation after slow volume loading appears to allow about half of the $18 \%$ extra $\mathrm{BV}$ to be accomodated in the veins. Interestingly, in the NP rats, $\mathrm{CO}$ had increased by 32 and $47 \%$ after rapid and slow volume loading, respectively, also with a twice as large increase in BV after the latter $(20$ versus $2 / 18.9 \mathrm{~mL}$ or $\approx 11 \%$ ). The larger rise in $\mathrm{CO}$ in NP rats after slow volume loading could indicate a lower capacity of the venous compliance to increase in response to slow volume loading in the NP state than in the P state. However, it is not possible to draw any conclusions on this matter on the basis of the (x-ray) methodology used in the present study.

Our values on baseline $\mathrm{CO}, \mathrm{CO}$ distribution, and organ flows in the NP rats agreed well with reported (microspheres) flow data in rat (19). Comparable data from normal and awake $P$ rats are scarce. A study in hypertensive rats confirmed our observation that $\mathrm{CO}$ in $\mathrm{P}$ rats is higher than in NP rats (23). The extra $\mathrm{CO}$ generated by slow volume loading was directed toward kidneys, carcass, and, in NP rats, also toward intestines (Table 4). The 
Table 3. Central hemodynamics, systemic shunting (fraction of injected microspheres recovered from the lungs), and regional blood flows ( $\mathrm{mL} / \mathrm{min}$ ) at baseline and after slow volume loading (VL) as observed in experiment 2

\begin{tabular}{lccccc}
\hline & \multicolumn{2}{c}{$\mathrm{P}$} & & \multicolumn{2}{c}{$\mathrm{NP}$} \\
\cline { 2 - 3 } \cline { 5 - 6 } & Baseline & Slow VL & & Baseline & Slow VL \\
\hline CO (mL/min) & $109 \pm 20$ & $155 \pm 38^{*}$ & & $99 \pm 19$ & $141 \pm 31^{*}$ \\
MAP (mm Hg) & $77 \pm 12$ & $71 \pm 17$ & & $102 \pm 24$ & $98 \pm 27^{*}$ \\
HR (bpm) & $404 \pm 40$ & $374 \pm 51$ & $356 \pm 47$ & $346 \pm 33$ \\
Systemic shunting (\%) & $4.0 \pm 1.6$ & $2.6 \pm 0.9$ & $2.5 \pm 2.4$ & $1.6 \pm 1.2$ \\
Blood flows (mL/min) to & & & & \\
$\quad$ Brain & $2.4 \pm 0.6$ & $3.0 \pm 1.1$ & $2.7 \pm 0.7$ & $4.5 \pm 1.2$ \\
Kidneys & $12 \pm 3$ & $20 \pm 7^{*}$ & & $13 \pm 5$ & $23 \pm 9^{*}$ \\
Carcass & $27 \pm 10$ & $41 \pm 13^{*}$ & & $33 \pm 10$ & $41 \pm 9^{*}$ \\
Intestines & $28 \pm 6$ & $37 \pm 15$ & $28 \pm 10$ & $38 \pm 13^{*}$ \\
$\quad$ Skin & $9 \pm 3$ & $10 \pm 4$ & $8 \pm 3$ & $8 \pm 3$ \\
$\quad$ Myometrium & $2.3 \pm 0.7$ & $1.7 \pm 0.6$ & $0.3 \pm 0.1 \dagger$ & $0.3 \pm 0.1$ \\
Placental blood flow & $2.2 \pm 1.0$ & $1.2 \pm 0.6^{*}$ & & \\
\hline
\end{tabular}

${ }^{*} p<0.05$ (Wilcoxon signed rank test).

$\dagger p<0.05$ (Mann-Whitney).

Table 4. Percentage change in organ flows after slow volume

\begin{tabular}{lcc}
\multicolumn{3}{c}{ loading } \\
\hline & $\mathrm{P}$ & $\mathrm{NP}$ \\
\hline Kidneys & +76 & +87 \\
Carcass & +81 & +28 \\
Intestines & $\mathrm{NS}$ & +37 \\
Placentas & -40 & \\
\hline
\end{tabular}



Fig. 3. The IVC outline as visualized by $\mathrm{A} \leftrightarrow \mathrm{P} x$-ray imaging in a 19$\mathrm{d} P$ rat pair. The rats are positioned in dorsal recumbency. Left panel, baseline; right panel, after $2 \mathrm{~h}$ of slow volume loading.

higher renal blood flow complies with the increased requirement for fluid dissipation. However, it is obscure why the blood flow toward skeletal tissues (carcass) and, in NP rats, also intestines had increased. These tissues are tightly autoregulated, and on the basis of the animals' behavior during slow volume loading (quietly sitting, little or no food intake), it seemed unlikely that nutritional flow requirements of these tissues had increased. Furthermore, it is unlikely that the observed fall in Hct had triggered a higher muscle (and in NP rats, intestinal) flow, inasmuch as studies in guinea pig have shown that isovolemic hemodilution does not increase the perfusion of these tissues (24). On the other hand, half of the resting blood flow to rabbit

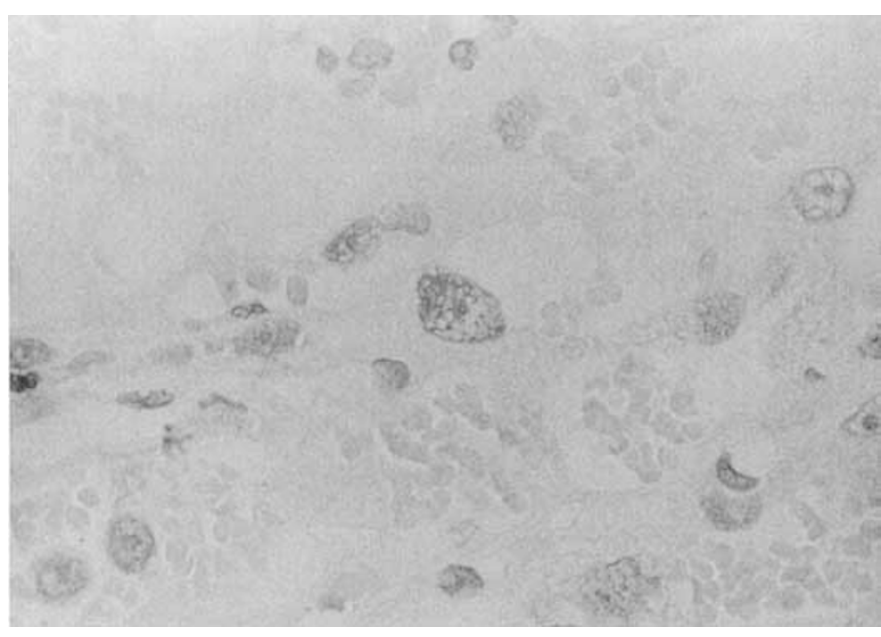

Fig. 4. Placental histology of $19-d \mathrm{P}$ rat in baseline condition $(\times 160)$. Small clusters of $\mathrm{RBC}$ in both fetal and maternal vessels alternative with cell-free areas.

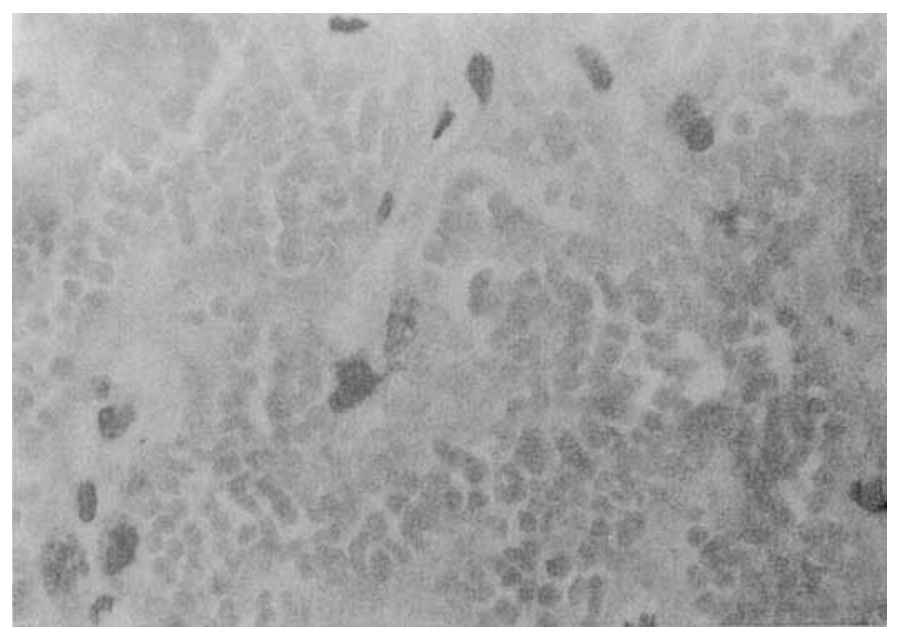

Fig. 5. Placental histology of 19-d P rat after $2 \mathrm{~h}$ of slow volume loading at $6 \mathrm{~mL} / \mathrm{h}(\times 160)$. Maternal lacunas show marked RBC crowding causing compression of fetal trabeculae.

Table 5. Hct, body weight, water intake, and urine production in basal conditions and after 24-h thirsting and weight loss and change in $B V$ caused by thirst period

\begin{tabular}{lccccc}
\hline & \multicolumn{2}{c}{ ThP/exp } & & \multicolumn{2}{c}{ ThNP/exp } \\
\cline { 2 - 3 } \cline { 5 - 6 } & Baseline & 24-h thirst & & Baseline & 24-h thirst \\
\hline Hct (vol\%) & $43.6 \pm 1.6$ & $46.6 \pm 1.9^{*}$ & & $48.8 \pm 1.9 \dagger$ & $52.2 \pm 1.6^{*}$ \\
Weight $(\mathrm{g})$ & $305 \pm 40$ & $290 \pm 43^{*}$ & & $254 \pm 14 \dagger$ & $238 \pm 16^{*}$ \\
$\begin{array}{l}\text { Water intake } \\
\quad(\mathrm{mL} / \mathrm{d})\end{array}$ & $37 \pm 7$ & 0 & & $26 \pm 5 \dagger$ & 0 \\
$\begin{array}{c}\text { Urine output } \\
\quad(\mathrm{mL} / \mathrm{d})\end{array}$ & $18 \pm 7$ & $4 \pm 2^{*}$ & & $14 \pm 5$ & $10 \pm 3$ \\
$\begin{array}{l}\text { Weight loss }(\mathrm{g}) \\
\text { BV change }(\%)\end{array}$ & & $-16 \pm 7$ & & $-19 \pm 3$ \\
\hline
\end{tabular}

${ }^{*} p<0.05$ (Wilcoxon signed rank test).

$\dagger p<0.05$ (Mann-Whitney).

tenuissimus muscle was found to be shunted through 5 to 11 $\mu \mathrm{m}$ arteriovenous shunts, and this "reserve" flow is redirected to the capillary bed during exercise $(25,26)$. In the intestinal bed, comparable functional arteriovenous shunts have been demonstrated (27).

Assuming unchanged nutritional flow in our study, it is well possible that the observed fall in vascular resistance in these 
Table 6. Hemodynamics in baseline and in volume-depleted (V-depleted) state*

\begin{tabular}{|c|c|c|c|c|c|c|}
\hline & \multicolumn{2}{|c|}{ ThP/exp } & \multicolumn{2}{|c|}{ NonthP/contr } & \multicolumn{2}{|c|}{ ThNP-exp } \\
\hline & Baseline & V-depleted & Baseline & Sham-V-depleted & Baseline & V-depleted \\
\hline $\mathrm{CO}(\mathrm{mL} / \mathrm{min})$ & $105 \pm 34$ & $96 \pm 21$ & $104 \pm 27$ & $109 \pm 19$ & $97 \pm 24$ & $94 \pm 29$ \\
\hline MAP (torr) & $107 \pm 13$ & $96 \pm 23$ & $90 \pm 7$ & $89 \pm 18$ & $104 \pm 13$ & $103 \pm 19$ \\
\hline $\mathrm{SVR}(\mathrm{mm} \mathrm{Hg} \cdot \mathrm{min} / \mathrm{mL})$ & $1.1 \pm 0.3$ & $1.0 \pm 0.3$ & $1.1 \pm 0.5$ & $0.9 \pm 0.3$ & $1.3 \pm 0.4$ & $1.2 \pm 0.4$ \\
\hline $\mathrm{HR}(\mathrm{bpm})$ & $413 \pm 29$ & $411 \pm 26$ & $400 \pm 51$ & $450 \pm 22$ & $403 \pm 33$ & $411 \pm 34$ \\
\hline $\mathrm{SV}(\mu \mathrm{L})$ & $259 \pm 95$ & $236 \pm 61$ & $265 \pm 109$ & $241 \pm 35$ & $242 \pm 62$ & $234 \pm 91$ \\
\hline
\end{tabular}

* None of the variables listed in this table changed significantly in response to thirsting as determined by the Wilcoxon signed rank test.

Table 7. Systemic shunting (defined as \% microspheres recovered from lungs) and regional blood flows ( $\mathrm{mL} / \mathrm{min})$ in baseline and in volume-depleted state ( $V$-depleted)

\begin{tabular}{|c|c|c|c|c|c|c|}
\hline & \multicolumn{2}{|c|}{ ThP/exp } & \multicolumn{2}{|c|}{ nonthP/contr } & \multicolumn{2}{|c|}{ ThNP/exp } \\
\hline & Baseline & V-depleted & Baseline & V-depleted & Baseline & V-depleted \\
\hline Systemic shunting & $5.9 \pm 2.8$ & $2.7 \pm 0.7^{*}$ & $5.8 \pm 2.3$ & $1.7 \pm 1.6^{*}$ & $2.5 \pm 2.1$ & $1.4 \pm 0.7$ \\
\hline \multicolumn{7}{|l|}{ Organ flows } \\
\hline Brain & $3 \pm 1$ & $3 \pm 1$ & $3 \pm 1$ & $3 \pm 1$ & $3 \pm 1$ & $3 \pm 2$ \\
\hline Kidneys & $11 \pm 4$ & $11 \pm 2$ & $13 \pm 5$ & $12 \pm 4$ & $16 \pm 5$ & $13 \pm 4$ \\
\hline Intestines & $26 \pm 9$ & $20 \pm 3$ & $24 \pm 10$ & $24 \pm 8$ & $26 \pm 6$ & $17 \pm 7$ \\
\hline Carcass & $31 \pm 10$ & $32 \pm 14$ & $23 \pm 9$ & $32 \pm 8$ & $30 \pm 10$ & $42 \pm 15$ \\
\hline Skin & $11 \pm 4$ & $8 \pm$ & $11 \pm 2$ & $11 \pm 3$ & $8 \pm 3$ & $7 \pm 3$ \\
\hline Myometrium & $2.4 \pm 1.3$ & $1.7 \pm 0.4$ & $2.3 \pm 1.1$ & $1.9 \pm 0.8$ & $0.3 \pm 0.1$ & $0.3 \pm 0.2$ \\
\hline Placental flow & $1.4 \pm 1.1$ & $4.0 \pm 1.8^{*}$ & $0.7 \pm 0.5$ & $4.4 \pm 1.1^{*}$ & & \\
\hline
\end{tabular}

$* p<0.05$ (Wilcoxon signed rank test).

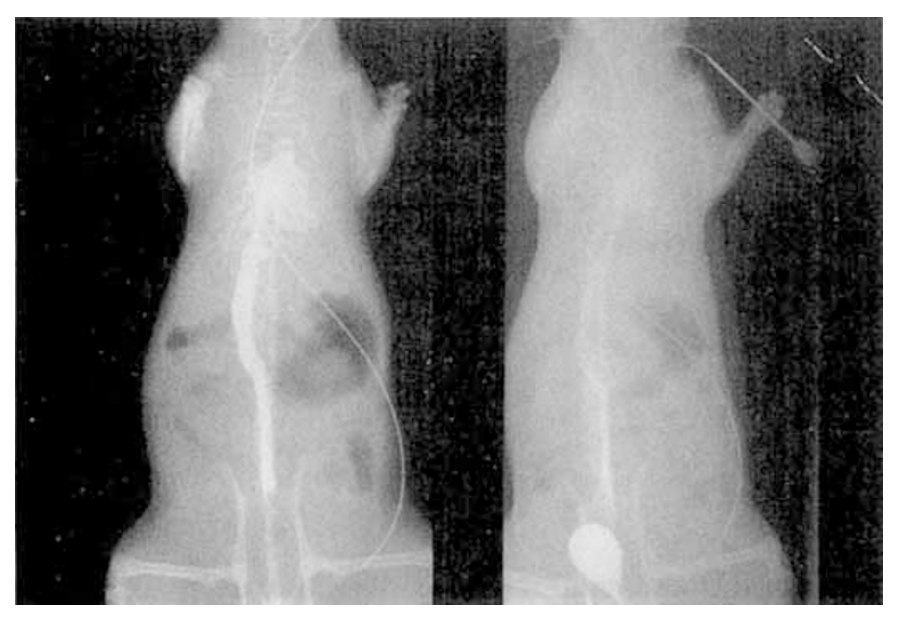

Fig. 6. The IVC outline as visualized by $A \leftrightarrow P$ X-ray in an NP rat pair. The rat on the left had free access to water before imaging and served as control for the rat on the right, which was thirsted during the preceding $24 \mathrm{~h}$.

tissues was caused by massive opening of these small shunts, a response that remains undetected by the microsphere method as applied in this study. Assuming that shunt recruitment occurs as part of the hemodynamic response to volume load, the sequence of events triggered by slow volume loading may be as follows: Gradually accumulating excess volume in the vascular bed immediately activates volume regulatory receptors in the venous system and atria, thus triggering the rise in renal blood flow and accelerated diuresis. The large capacitance vessels dilate gradually, accomodating as much excess volume as possible. If the capacity of the large veins to dilate is exceeded, MCFP increases and causes a rise in venous return $(20,21)$. As a consequence, a rise in $\mathrm{CO}$ in excess of nutritional demands is generated. This excess systemic flow is subsequently shunted across the systemic circulation through small arteriovenous shunts in skeletal tissues and, in NP rats, intestines. Massive arteriovenous shunting, possibly in combination with altered arteriolar vasomotion activity (28), may represent an efficient mechanism to protect the Starling forces in the capillary bed.
In $\mathrm{P}$ rats, slow volume loading caused a consistent decrease in placental blood flow. If the $\mathrm{CO}$ rise in excess of nutritional flow demands is assumed to be caused by a rise in MCFP, it is conceivable that the flow decrease, which seems to occur selectively in the placentas, is a direct consequence of the unique microarchitecture of the rat placenta. The hemotrichorial placenta of this species differs from all other vascular beds by its lack of lymphatic drainage, absent venules, and peculiar rectangular branching pattern in the microcirculation without possibility for capillary and/or shunt opening or closure. Particularly the latter feature implies that a fall in perfusion pressure associated with a rise in MCFP can be expected to reduce local shear forces. A further reduction of already low shear forces will compromise rheologic conditions, causing red cell sludging and accelerated formation of red cell aggregates (Fig. 5).

Support for this concept was expected to come from the subsequent studies on the hemodynamic response to slow volume depletion. In these studies the finding of a hemodynamic response opposite to the one observed after slow volume loading would provide support for the proposed mechanisms delineated above. In an attempt to minimize the activation of the sympathetic nervous system, volume depletion was induced both gradually and mildly by $24-\mathrm{h}$ thirsting. The weight loss of $16-19 \mathrm{~g}$ in the ThP/exp and ThNP/exp groups was probably only partly caused by loss of body water, inasmuch as the rats also tended to eat less during the period of thirsting. In this context, it should be emphasized that the absolute weight loss in the ThP/exp rats was probably larger because their normal daily weight gain in late pregnancy $(\approx 20 \mathrm{~g})$ was reversed. P rats also drink more than NP rats (Table 5), and the observed $7 \%$ volume depletion develops while the $\mathrm{P}$ rat is in the process of expanding its BV. Therefore, these results confirm previous data (29) that indicate that 24-h thirsting has a larger impact on the $\mathrm{P}$ rat than on the NP rat. In the ThP/exp and ThNP/exp groups, none of the hemodynamic parameters changed significantly, despite a $7 \%$ decrease in BV. CO and all organ flows were maintained, which indicates that the observed venoconstriction (Fig. 6) was apparently adequate to preserve venous return. Placental blood flow had increased in the ThP/exp group. Comparison of the postthirst value in $\mathrm{ThP} / \mathrm{exp}$ with the second blood flow value in the $\mathrm{ThP} /$ control group indicated that the rise in placental blood flow 
was entirely explained by the advancement in pregnancy. Unfortunately, the decrease in the size of the venous compartment could not be quantified with the method used. The similarity in $\mathrm{CO}$ rise in $\mathrm{P}$ rats after rapid and slow volume loading, despite a twice as large increase in $\mathrm{BV}$ after the latter, suggested that the venous compartment could accommodate about $10 \%$ extra volume. If the venous compartment also has the capacity to neutralize volume reductions of up to $10 \%$, the $7 \% \mathrm{BV}$ depletion observed after 24-h thirsting may have been too small to elicit a "complete" hemodynamic response, which theoretically would include, besides venoconstriction, a fall in renal blood flow and $\mathrm{CO}$.

With respect to possible clinical implications, one should realize that 24-h thirsting induces volume depletion characterized by a certain rate, magnitude, and duration. The magnitude of the volume depletion induced in the present study $(7 \%)$ is likely to be smaller than that occurring in preeclamptic pregnancies, where BV may be reduced by as much as $30 \%(30,31)$. In pregnancies in which volume depletion has developed to such an extent, venoconstriction may be inadequate to maintain venous return. One may speculate that the resulting inadequacy of $\mathrm{CO}$ has led to an increased sympathetic tone and, thus, to a redistribution of $\mathrm{CO}$ at the expense of kidneys and possibly also placenta. However, this concept assumes a yet unproved causal role of volume depletion in the pathogenesis of this disease.

\section{REFERENCES}

1. Lees MM, Taylor SH, Scott DB, Kerr MG 1967 A study of cardiac output at rest throughout pregnancy. J Obstet Gynaecol Br Commonw 74:319-328

2. Pirani BBK, Campbell DM, MacGillivray I 1973 Plasma volume in normal first pregnancy. J Obstet Gynaecol Br Commonw 80:884-887

3. Capeless EL, Clapp JF 1989 Cardiovascular changes in early phase of pregnancy. Am J Obstet Gynecol 161:1449-1453

4. Clapp JF, Seaward BL, Sleamaker RS, Hiser J 1988 Maternal physiologic adaptations to early human pregnancy. Am J Obstet Gynecol 159:14561460

5. Hilton JG, Kanter DM, Hays DR, Bowen EH, Golub JR, Keatings JH 1955 The effect of acute arteriovenous fistulae on renal functions. J Clin Invest $34: 732$

6. Haneda K, Hessel II EA, Thomas R, Huntsman LL 1986 Cardiovascular response to rapid infusion of lactated Ringer's. Tohoku J Exp Med 149:397406

7. Prather JW, Taylor AE, Guyton AC 1969 Effect of blood volume, mean circulatory pressure, and stress relaxation on cardiac output. Am J Physiol 216:467-472

8. Goodlin RC, Quaife MA, Dirksen JW 1981 The significance, diagnosis, and treatment of maternal hypovolemia as associated with fetal/maternal illness. Semin Perinatol 5:163-174

9. Wallenburg HCS 1988 Hemodynamics in hypertensive pregnancy. In: Rubin PC (ed) Handbook of Hypertension, Vol 10, Hypertension in Pregnancy. Elsevier, Amsterdam, pp 68-101
10. Alexander RS 1956 Reflex alterations in venomotor tone produced by venous congestion. Circ Res 4:49-53

11. Porciuncula CI, Armstrong GG, Guyton AC, Stone HL 1964 Delayed compliance in the external jugular vein of the dog. Am J Physiol 207:728-732

12. Atherton JC, Dark JM, Garland HO, Morgan MRA, Pidgeon J, Soni S 1982 Changes in water and electrolyte balance, plasma volume and composition during pregnancy in the rat. J Physiol (Lond) 330:81-93

13. Simchon S, Manger WM, Carlin RD, Peeters LL, Rodriguez J, Batista D, Brown T, Merchant NB, Jan K-M, Chien S 1989 Salt-induced hypertension in Dahl salt-sensitive rats. Hemodynamic and renal responses. Hypertension 13:612-621

14. Smits IFM, Coleman TG, Smith TL, Kasbergen CM, Van Essen H, StruykerBoudier HAJ 1982 Antihypertensive effect of propanolol in conscious spontaneously hypertensive rat. J Cardiovasc Pharmacol 4:903-914

15. Davies MJ 1987 Polygeline. Dev Biol Stand 67:129-131

16. Lowe KC, McNaughton DC 1984 Acute physiological responses during blood substitution with colloidal gelatin in conscious rats. Exp 40:700-701

17. Peeters LH, Grutters G, Martin CB 1980 Distribution of cardiac output in the unstressed pregnant guinea pig. Am J Obstet Gynecol 138:1177-1184

18. Saxena PR, Schamhardt HC, Forsyth RP, Loeve J 1980 Computer programs for the radioactive microsphere technique. Determination of regional blood flows and other hemodynamic variables in different experimental conditions. Comput Program Biomed 12:63-84

19. Ishise S, Pegram BL, Yamamoto J, Kitamura J, Frohlich Y 1980 Reference sample microspheres method: cardiac output and blood flows in conscious rat. Am J Physiol 239:H443-H449

20. Rothe CF 1986 Physiology of venous return. Arch Intern Med 146:977-982

21. Rothe CF 1983 Reflex control of veins and vascular capacitance. Physiol Rev 63:1281-1342

22. Ludbrook J, Graham WF 1984 The role of cardiac receptor and arterial baroreceptor reflexes in control of the circulation during acute change in blood volume in the conscious rabbit. Circ Res 54:424-435

23. Ahokas RA, Anderson GD, Lipshitz J 1983 Cardiac output and uteroplacental blood flow in diet-restricted and diet-repleted pregnant rats. Am $\mathrm{J}$ Obstet Gynecol 146:6-13

24. Peeters LLH, Verkeste CM, Saxena PM, Wallenburg HCS 1987 Hemodynamic effects of isovolemic hemodilution and hemoconcentration in the awake late-pregnant guinea pig. Pediatr Res 21:584-589

25. Oude Vrielink HHE, Slaaf DW, Tangelder GJ, Reneman RS 1987 Does capillary recruitment exist in young rabbit skeletal muscle? Int $J$ Microcirc Clin Exp 6:321-332

26. Lindbom L, Arfors KE 1984 Non-homogeneous blood flow distribution in the rabbit tenuissimus muscle. Acta Physiol Scand 122:225-233

27. Lundgren O 1984 Microcirculation of the gastrointestinal tract and pancreas. In: Renkin EM, Michel CC (eds) Handbook of Physiology: The Cardiovascular System, Vol 4, Part 2. Williams \& Wilkins, Baltimore, pp 799-864

28. Iida $N 1990$ Effects of vasomotion and venous pressure elevation on capillarytissue fluid exchange across hetero-porous membrane. Biorheology 27:205224

29. Durr JA, Stamoutsos B, Lindheimer MD 1981 Osmoregulation during pregnancy in the rat. J Clin Invest 68:337-346

30. Gallery EDM, Hunyor SN, Gyory AZ 1979 Plasma volume contraction: significant factor in both pregnancy-associated hypertension preeclampsia and chronic hypertension in pregnancy. Q J Med 48:593-602

31. Chesley LC, Lindheimer MD 1988 Renal hemodynamics and intravascular volume in normal and hypertensive pregnancy. In: Rubin PC (ed) Handbook of Hypertension, Vol 10, Hypertension in Pregnancy. Elsevier, Amsterdam, pp 38-65 